\title{
EDUCACIÓN, EXPERIENCIA Y PEDAGOGÍA BIOPOLÍTICA. POR UN DIAGNÓSTICO DE NUESTRO PRESENTE EDUCACIONAL
}

\author{
Fernando Bárcena Orbe \\ Pedro Angelo Pagni** \\ Rodrigo Pelloso Gelamo***:
}

\section{Marco teórico}

El presente proyecto de colaboración aspira a un intercambio de experiencias de investigación en relación con una temática que en la actualidad se inscribe en diversas tentativas de investigación de carácter multidisciplinar, y que busca explorar las consecuencias políticas, sociales y educativas del fenómeno biopolítico, temática que en la actualidad está siendo objeto de gran atención por diversos investigadores pertenecientes al campo de las ciencias humanas y sociales.

En efecto, las discusiones en torno a la biopolítica se han intensificado en los últimos años, en un intento de comprender los acontecimientos políticos que fuerzan una revisión de los fundamentos de la teoría política y que afectan de una forma notoria al diseño de diversas políticas educativas. En este sentido, el marco teórico de este proyecto de colaboración incorpora los estudios que se han venido realizando, en las últimas décadas, a partir de la reflexión de Hannah Arendt (1951) sobre el «pensamiento de la raza» y el totalitarismo; de los escritos y cursos sobre la «población» dictados por Michel Foucault en el Collége de France (Foucault, 1976, 1997, 2006 y 2004), así como por otros autores de prestigio, como Giorgio Agamben (1998), Toni Negri, Roberto Esposito (2003, 2005, 2006, 2008 y 2009), Simona Forti (2006 y 2008) y Alain Brossat (1996, 2000 y 2003).

Siguiendo los estudios, pioneros en este campo, de Michel Foucault —que usaba como términos sinónimos los conceptos de «biopoder» y «biopolítica», aunque finalmente se ha impuesto este último - «habría que hablar de 'biopolítica'

\footnotetext{
* Doutor em Filosofia da Educação. Professor da Universidad Complutense de Madrid.

** Doutor em Educação. Professor do Departamento de Administração e Supervisão Escolar da Universidade Estadual Paulista Julio de Mesquita Filho - Campus Marilia.

*** Doutor em Educação. Professor do Departamento de Didática da Universidade Estadual Paulista Julio de Mesquita Filho - Campus Marília.
}

ORBE, Fernando Bárcena; PAGNI, Pedro Angelo; GELAMO, Rodrigo Gellamo. Educación, experiencia y pedagogía biopolítica. Por un diagnóstico de nuestro presente educacional. Revista Sul-Americana de Filosofia e Educação. Número 20: maio-out/2013, p. 87-106. 
para designar lo que hace entrar a la vida y sus mecanismos en el dominio de los cálculos explícitos y convierte al poder-saber en un agente de transformación de la vida humana» (Foucault, 2002, 173). Según esto, el objeto de la biopolítica es la vida, entendida, en sentido griego, como mera zoé - es decir, el «simple hecho de vivir», que es común a todos los seres vivos-, vida distinta del bíos, que indica la forma o manera de vivir propia de un individuo o grupo humano (como vida narrable y experimentada como humana, en un sentido distintivamente biográfico). Bajo un primer análisis, el objeto de la moderna biopolítica es gobernar, administrar y normalizar la vida del individuo y las poblaciones. La biopolítica aspira a un gobierno de una población, de la que extrae los mayores beneficios políticos, económicos y en un cierto orden pedagógico, porque para su éxito se requiere educar las conciencias de los individuos de modo que comprendan las ventajas de dicha administración.

Se parte de la hipótesis de que los procesos de modernización de los sistemas educativos -en los procesos de enseñanza y aprendizaje, dentro de los contextos formales de educación - presenta un carácter ambivalente, al verse los mismos, simultáneamente, impulsados por una operación normalizadora característica central de la modernidad biopolítica, o lo que Bernard Stiegler denomina psicopoder (Stiegler, 2008) - pero generando un deterioro de la experiencia formativa de los sujetos de la educación en el seno de la sociedad del conocimiento y de la información.

Indudablemente, un análisis equilibrado del fenómeno biopolítico, con respecto a sus efectos en la biografía formativa de los individuos, nos lleva a decir que no todo en él es negativo; es indudable que la biopolítica, ligada como está al desarrollo de las tecnologías, permite combatir mejor las enfermedades, aumenta la esperanza de vida de la población y permite que los Estados dediquen mayores recursos al capítulo del "gasto social", desde la educación hasta el cuidado de las "personas dependientes" (Ugarte Pérez, 2005; Mendiola Gonzalo, 2009); ejemplos de ello son, en España, la aprobación durante el último gobierno socialista de la "ley de dependencia", ley que cubre de hecho un vacío legal, pero que, al carecer de dotación económica, la vuelve, en el fondo, ineficaz; y en Brasil, análogamente 
a lo que ocurre en el caso español, también puede hablarse de una serie de políticas de asistencia social que tratan de integrar una parte significativa de la población que viven en un estado de miseria encaminadas a proporcionar condiciones para su acceso a los bienes de consumo tornándose, así, ciudadanos, así como políticas educativas que buscan permitir que, personas que no reúnen las condiciones para permanecer en el proceso educativo, puedan acceder a una Universidad pública o costearse un curso privado.

La novedad investigadora del programa de investigación que se pretende aportar a los actuales estudios biopolíticos en el campo de las ciencias humanas y sociales —y cada vez más presentes tanto en las universidades brasileñas como españolas-, consiste en una lectura pedagógica de este fenómeno, una reflexión que, desde esta perspectiva de análisis, hasta el momento no ha sido realizada ni sistematizada suficientemente. De ahí el énfasis que se ha querido poner en la categoría de pedagogía biopolítica. ${ }^{1}$

Diversos factores invitan a considerar críticamente los efectos que dicho proceso de modernización de la educación tiene en toda tentativa de configurar una pedagogía de la experiencia; entre otros, los siguientes: el acento que los sistemas educativos modernos han puesto en las finalidades educativas de índole ético-social; la intensidad de los debates sociales y académicos en torno al lugar que en las sociedades pluralistas ocupan visiones trascendentes de la vida humana y la formación del hombre; y el lugar cada vez más nuclear que las legislaciones jurídico-políticas prestan a un concepto no siempre claro de «calidad de vida humana» (Brossat, 2010). Pero lo que nos interesa destacar aquí es otro asunto. En nuestra contemporaneidad educativa se está produciendo una inversión de la jerarquía de las generaciones que destruye al mismo tiempo la singularidad de sus diferencias, organizando su plena confusión. Los medios específicos de control de la atención, tanto psíquica como social, de los jóvenes, a través de los aparatos

\footnotetext{
${ }^{1}$ La abundancia de estudios sobre la biopolítica moderna, desde el punto de vista de uma reflexión filosófica, contrasta con la escasa atención que se ha prestado a esta cuestión en el campo de la teoría y la filosofía de la educación. Entre ellos, cabe destacar: Bárcena, F.; Asensio, J. M; Mèlich, J. C. (2001); Bárcena, F. (2008); Bárcena, F. (2006); Bárcena, F. (2009); Lewis, T. E. (2009); Lewis, T. E. (2007); Lewis, T. E. (2006); Gadelha (2010); Dalbolsco (2009); Pagni (2010a); Bueno (2009).
} 
propios de la sociedad de las telecomunicaciones, promueven una minorización del adulto (por su desresponsabilización frente a los jóvenes) y la constitución del joven como prescriptor del adulto de sus propias pulsiones, a través de una operación de falsa adultización.

Un ejemplo de ello (sin duda aplicable a muchos otros países) puede ser la legislación penal francesa, discutida y aprobada en el año 2007, que prescribía para ciertos delitos cometidos por jóvenes, en caso de recidiva-, que los mismos no serían juzgados en función de su minoría de edad, sino que la ley les sería aplicada como a los adultos, mayores de edad. Como justificación de este importante cambio legislativo se decía que la disposición jurídica que limitaba la edad de responsabilidad penal conllevaba un sentimiento de impunidad. Con esta disposición legal, al mismo tiempo que se cuestiona la minoría de los jóvenes delincuentes, se pone en causa la mayoridad de sus ascendientes adultos, liberándose con ello su responsabilidad como tales. Esta medida, en definitiva, tiene importantes consecuencias para un pensamiento de la educación, en el sentido en que ésta es precisamente la encargada de acompañar a los jóvenes hacia la responsabilidad, es decir, a un estado de mayoría de edad. Al mayorizar al joven, fuera de tiempo, y desresponsabilizar al adulto, infantilizándolo, se invierten los términos y se ocasiona un estado de indiferenciación entre las generaciones. Otro ejemplo del mismo fenómeno es la campaña que, por aquellas mismas fechas, promovió el canal televisivo dedicado al mundo infantil y juvenil "Canal J", cuyo eslogan de base, sobre el fondo de una fotografía en la que frente a un niño perfectamente aburrido aparecía su abuelo incapaz ya de hacerle reír y entretenerle, era el siguiente: Les enfants méritent mieux que ça. Ils méritent Canal J. De forma clara, esta campaña supone una intrusión de los mecanismos psicotécnicos de control de la atención en los procesos de transmisión intergeneracional. Como algunos investigadores ya han planteado, las generaciones más jóvenes -infancia y adolescencia—, entendidas como formas de relación social, constituyen hoy por hoy una preocupación biopolítica fundamental, así como el diseño controlado de sus ritmos de aprendizaje, enfocados al desarrollo de competencias definidas por el mercado de trabajo (Bustelo, 2007). 
Al destacar como un rasgo central de nuestro tiempo la condición biopolítica, aspiramos a establecer, en relación con los mecanismos que activa y pone en marcha -y en sus efectos en la teoría y la práctica educativas-, una relación que llamaremos de contemporaneidad. Adoptamos aquí el sentido, acuñado por Agamben, de lo contemporáneo, según el cual "pertenece verdaderamente a su tiempo, es realmente contemporáneo, aquél que no coincide perfectamente con él ni si adapta a sus pretensiones, y es por ello, en este sentido, inactual; pero justamente por ello, justamente a través de esta diferencia y de ese anacronismo, es capaz más que los demás de percibir y de entender su tiempo" (Agamben, 2008). Lo contemporáneo, en primer lugar, establece una relación intempestiva con el presente, es decir, una relación de distanciamiento y extrañamiento con el propio tiempo. Y, en segundo término, en esta relación se identifica como un cierto mal algunos elementos configuradores de los que la propia época se siente más orgullosa. Por tanto, ser contemporáneo es aprender a mirar el tiempo presente para detectar las zonas de sombra del presente que una excesiva exposición lumínica impediría percibir (Didi-Huberman, 2009). Con eso nos imponemos una tarea: la de diagnosticar en nuestro presente los elementos o factores que nos impiden hacer una experiencia en y de la contemporaneidad. De esta manera, el tema en cuestión se justifica en la medida en que el empobrecimiento de la experiencia - la capacidad de pensarla y narrarla, observada en nuestros días-, constituye un importante problema para el pensamiento de la educación, y también para su puesta en marcha y realización.

Walter Benjamin (2000a) fue uno de los primeros filósofos en diagnosticar ese problema y sus consecuencias para la vida humana. Theodor Adorno (1992) parece haber radicalizado ese diagnóstico sobre el empobrecimiento de la experiencia, aunque, según Giorgio Agamben (2001), el proyecto benjaminiano lo caracteriza de un modo más próximo a lo que vivimos en nuestros días. Agamben argumenta que no necesitamos presenciar ninguna catástrofe o guerra para percibir la destrucción de la experiencia en nuestros días. Finalmente, lo cotidiano del hombre contemporáneo no contiene casi nada que pueda ser traducible en 
términos de experiencia: ni el trabajo, ni las noticias de los periódicos, ni los viajes, ni el consumo ni, podríamos agregar a la lista, la vida escolar.

Este problema forma parte de la crítica de la problematización filosóficopedagógica en buena parte de la filosofía contemporánea, en relación con el proyecto de la modernidad. Esa crítica consiste en recordar a ese proyecto los costes de su desarrollo, en términos de reducción de la experiencia a lo empírico (experimentum), de restricción del pensamiento al conocimiento científico y a la tecnología, algo parecido a lo que acontece con el silenciamiento del individuo ante un vacío existencial sin precedentes. La crítica de la modernidad se ha erigido en una de las tareas primordiales de las filosofías y de las teorías sociales contemporáneas, lo que las ha conducido, no sólo a recordar este empobrecimiento de la experiencia y su consiguiente pérdida de capacidad narrativa, sino también a la necesidad de pensar el tiempo presente de otro modo, para así poder discutir sus consecuencias pedagógicas desde el punto de vista de una filosofía de la educación.

Siguiendo la interpretación de Agamben (1998), puede decirse que el vacío existencial presentido por todos, y que las nuevas generaciones experimentan hoy en día, es la resultante de la inclusión de la nuda vida (zoé) - la vida meramente biológica- en los mecanismos y cálculos del poder estatal y de transformación de la política clásica en biopolítica, entendiendo este proceso como un desarrollo casi natural de la modernidad. Recurriendo al pensamiento del último Foucault, el filósofo italiano considera que, en términos políticos, dicha transformación consiste en un disciplinamiento de la nuda vida que aspira a promover la subordinación de la población a los dispositivos del poder del Estado, y en docilizar los cuerpos para que los individuos se adapten a modos normalizados de existencia. Sin esta inclusión de la vida en los mecanismos y cálculos de poder, esa transformación de la política clásica en la sociedad moderna nunca se habría desarrollado en los términos en que lo hizo.

Con todo, más que formar parte de los mecanismos de poder, la nuda vida pasa a ocupar progresivamente el centro de la escena política en la modernidad. El derecho a la vida, que regula la biopolítica, pasó a desvirtuarse en los términos de 
una pregunta y de una definición sobre qué clase de vida merece la pena ser vivida, restringiéndose la vida a una mera supervivencia en el seno de los choques provocados por lo cotidiano. Bajo esas circunstancias, quien no se adaptase a un vivir, limitado ya a mera supervivencia, quien viviese de modo distinto a lo establecido por el poder soberano, pasaría a ser excluido de la esfera pública y a ser cercenado en sus modos de existencia; el Estado, entonces, reglamentaría la nuda vida, aunque inspirada en una racionalidad indiferente a la vida, entendida como bíos.

La paradoja señalada por Agamben puede percibirse no sólo en lo poco que quedó de auténtica esfera pública en las acciones del Estado, sino también en su distribución en las imágenes que extienden los medios de comunicación y en los discursos de las diversas artes de gobierno; $y$, entre ellos, en el discurso pedagógico. Bajo las condiciones de la llamada "sociedad del conocimiento", la misma hace de cierto "progresismo pedagógico" un eslogan según el cual la tarea prioritaria de la institución escolar es "enseñar a vivir". Ahora bien, este aprendizaje de la vida adquiere, en el contexto de la sociedad de aprendizaje, connotaciones y consecuencias muy específicas. El lema del discurso de la sociedad de aprendizaje es "aprender a aprender" ("formación continua" o "aprendizaje para toda la vida"), el cual coloca al ciudadano en la situación de una especie de eterno aprendiz. La categoría central de este discurso pedagógico es la del aprendizaje basado en competencias, quedando obsoleta la cuestión relativa a la transmisión de tradiciones vivas de pensamiento. Puede decirse, entonces, que la supervivencia, real y efectiva, desde el punto de vista social, del ciudadano, depende de una lógica de control pedagógico en relación con dicho aprendizaje, es decir: con el aprender a aprender (Masschelein, 2001). La lógica pedagógica biopolítica desplaza el aprender, como un momento autónomo focalizado en la experiencia de quien aprende, hacia un momento infantilizado donde se privilegian determinadas técnicas de control, normalización y gobierno de la formación, pedagógicamente capturada (Stiegler, 2008 y 2010).

Como hemos señalado, la figura del aprendiz eterno expresa adecuadamente esta racionalidad de la biopolítica. La biopolítica hace uso de 


\section{Revista Sul-Americana de Filosofia e Educação - RESAFE}

cualquier sistema o subsistema social —como el sistema educativo- para administrar, gestionar y normalizar las vidas de los ciudadanos en un marco cuyos valores centrales serán la seguridad, el orden, la jerarquía, la clasificación, la métrica, la cuadrícula. En este contexto, la idea de la educación como "formación" (Bildung, Paideia), el sentido de una experiencia educativa entendida desde el lenguaje de la experiencia, queda literalmente excluida de un discurso pedagógico biopolítico focalizado hacia la noción privilegiada de habilidades y competencias.

Con ese modo de racionalización del mundo, la gobernamentabilidad pedagógica desarrollada en la institución escolar contribuyó a extender su dominio en la formación de las nuevas generaciones y en los capilares de la subjetividad humana, interfiriendo en las esperanzas de ruptura con el pasado y desfigurando la experiencia del sujeto, tan necesarias como son para la transformación de la realidad y de uno mismo. Enmarañada en esa racionalización, su acción no parece ofrecer posibilidades de escape a la racionalidad totalizadora que la comprende. Su objetivo es reglamentar las resistencias emergentes en los espacios y tiempos escolares, prescribir normas de conducta y disciplinar los cuerpos de los destinatarios del discurso puestos en circulación, facilitando, de este modo, su incorporación a los modos dominantes de conformación de la praxis educativa. Esto acontece en la medida en que el arte pedagógico formaliza el aprendizaje y limita la enseñanza a una técnica instrumental, elaborándolos teóricamente bajo la óptica de una racionalidad que excluye aquello que proviene de lo sensible, de lo inefable, en fin, de la experiencia, y rompiendo los lazos de los saberes y de las prácticas escolares con la vida.

La racionalidad instrumental o técnica en la que se apoya la enseñanza, al limitarse a un ajuste de medios para fines dados — con vistas a una mayor eficiencia de la transmisión de lo que es enseñado y de la objetividad en la apreciación cognitiva por parte de sus destinatarios-, excluye la experiencia y la vida en la elaboración de los saberes y de las prácticas escolares. Los argumentos utilizados para justificar tal exclusión son los siguientes: 
(1) La experiencia escapa a la regularidad, la estabilidad y la determinación que toda planificación presuponen, así como a la lógica y a la determinación previa de los saberes y las prácticas escolares en la que se sustenta su organización.

(2) El aprender por la experiencia es algo externo a esa racionalidad que, en términos pedagógicos, se materializa en un conjunto de prácticas de enseñanza y se legitima en la articulación de una serie de saberes.

(3) La experiencia pone en riesgo la objetividad y la eficiencia de la performance técnica en que se convierte la praxis educativa en la actualidad.

Por ese motivo, a la hora de juzgar la experiencia en la actividad educativa, desde el punto de vista técnico, queda encuadrada en categorías previas, racionalmente elaboradas por una lógica y enunciadas discursivamente y reglamentada por un género cognitivo de lenguaje. Por más que el aprendizaje de la experiencia, en sus intersecciones con la vida, movilice géneros de lenguaje diferentes y diversos, no deja de estar subordinado a la elaboración estrictamente lógica: la enunciación discursiva y la reglamentación, características esenciales de lo cognitivo, dimensión privilegiada en la actividad de la enseñanza. De este modo, se procura minimizar pragmáticamente los efectos de la transmisión de conocimientos, substrayendo sus relaciones con la vida, a costa de una violencia simbólica ejercida sobre ella.

La cuestión de la transmisión es, entonces, de crucial importancia para este proyecto, pues es en ella donde cabe percibir la indignidad que la experiencia (en expresión de Benjamin) alcanzó en la relación pedagógica. Y esto debido a que la enseñanza pasó a privilegiar cierta transmisión abstracta del conocimiento, en el par información-recepción. La lógica de la enseñanza vigente orienta la relación enseñar/aprender a una única función: enseñar es transmitir las verdaderas representaciones del discurso docente y aprender se limita a asimilar adecuadamente lo que fue explicado, estableciéndose una correlación entre la explicación del profesor/ comprensión del alumno, para posteriormente repetir, de 
modo claro y distinto, lo aprendido. El profesor, presionado muchas veces por el miedo que las situaciones que se escapan a toda planificación genera en su actuación, y para las cuales carece de recursos, como no sean los meramente técnico-didácticos, se vuelve rehén de una lógica explicatoria, según la cual la enseñanza consiste en una transmisión reducida a la explicación (Rancière, 1987).

En esta lógica existe implícita una relación biopolítica, según la cual quien explica es el detentador de los conocimientos necesarios que le permitirán asumir la responsabilidad de transmitir los contenidos a quienes no los poseen, manteniendo una lógica normalizadora y reproductora de conocimientos. Con este tipo de enseñanza se privilegia la transmisión de un tipo de información, marcada por el saber técnico, cuyo objetivo es enseñar a reconocer la forma y el contenido de un determinado saber. Por medio de la estructura de la explicación, la enseñanza corre el riesgo de convertirte en una adecuación causal entre los que se aprende y lo que fue enseñado. O dicho en otros términos, en el acto de aprender la relación entre los alumnos y el contenido pasa a través de un proceso de instrumentalización del aprendizaje que reside en la explicación presentada por el profesor como una forma legitima de captar la verdadera significación de las ideas que les son presentadas a aquéllos. La explicación se vuelve un medio de "(in)formar" a los alumnos. Así, enseñar se reduce a una explicación que el profesor ofrece, administra, instrumentaliza y gestiona, con vistas a formar determinadas competencias en los alumnos. El mecanismo que da soporte a este proceso, la palabra (explicatoria) del profesor, rompe el supuesto mutismo (equivalente a una falta de comprensión) en el alumno, abriendo un hiato entre el mundo, lo que el profesor enseñó/transmitió y lo que el alumno ha de comprender.

La transmisión de una tradición de pensamiento a las nuevas generaciones, que ya desde hace mucho tiempo estaba normalizada por la fórmula enseñanza/aprendizaje, encuentra, actualmente, amparo metodológico en otra función: explicación/asimilación, cuyo objetivo es propiciar la acumulación de informaciones sobre determinados conocimientos que supuestamente serían capaces de generar las competencias y las habilidades esperadas por las políticas educativas. El problema que se puede observar en esa nueva formulación es que la 
explicación no está necesariamente amparada por la experiencia del profesor en su inscripción en una tradición viva de pensamiento que se reactualiza en el tiempo. De esta manera, por parte del alumno, la asimilación se constituye en una relación puramente cognitiva con la explicación conferida por el profesor y, por eso, muchas veces no supone una verdadera experiencia de pensamiento para él.

Desde esta perspectiva, la experiencia de pensamiento de los alumnos de nada valdría, pues lo que se espera de ellos es que adquieran una representación adecuada de los significados producidos y que logren relacionarlos con significantes ya previamente consolidados, con los saberes en circulación. Así, pensar, en este registro, se configura como un ejercicio de erudición vacío, desarrollado a partir del cúmulo de conocimientos adquiridos sobre las materias enseñadas, lo que dificulta una experiencia del pensamiento capaz de producir una fisura en la relación significativa dominante, permitiendo al "aprendiz" la búsqueda de una resignificación de su relación con lo adquirido por la tradición, con la vida y con el mundo.

En este sentido, los conocimientos que fueron producidos por el alumno en su relación con la tradición, o con su propia experiencia del pensamiento, en muchos casos son moldeados, o incluso negados, a partir de la explicación del profesor. La lógica explicatoria, entendida como una (bio)política de la enseñanza, silencia tanto la tradición como al aprendiz en su ejercicio de pensamiento, su propia experiencia del pensar, para dar voz a aquello que las políticas educativas afirman como necesario para el alumno, en términos de habilidades o competencias exigidas para su acceso en la sociedad y el mercado. Una consecuencia de todo ello es el rechazo, cuando no la franca imposibilidad, por parte del alumno, en mantener la tensión en el interior de los conflictos que el mismo pensamiento proporciona en su devenir, limitándose a asimilar la explicación correcta que satisfaga o acalle el conflicto singular y movilizador de las ideas. El problema, entonces, parece instalarse en la relación entre el conocimiento adquirido por medio de la transmisión/asimilación y el construido por la experiencia del pensamiento (o en la relación entre ambos) frente a la tradición 
cultural (que puede ser entendida, aquí, como elemento que da soporte a la Paidea o a la Bildung).

Tanto este problema, como los relacionados con la trasmisión en la enseñanza y con los de la atrofia de la experiencia en la acción educativa, han sido abordados, al menos, de dos modos distintos, que pueden ser atendidos aquí y reformulados: (1) De un lado, se pueden recuperar las cualidades estéticas comprendidas en la experiencia educativa y discutir si pueden ser traducidas $y / o$ transmitidas en la enseñanza, a partir del pensamiento reflexivo de los sujetos de la práctica pedagógica, desde el pragmatismo de John Dewey ${ }^{2}$, de la hermenéutica de Hans-Georg Gadamer o, incluso, de Jürgen Habermas ${ }^{3}$, con vistas a integrar su expresividad en los lenguajes constitutivos de los saberes y las prácticas escolares ${ }^{4}$; y (2) De otro, se pueden analizar las dimensiones estética, ética y política de la experiencia educativa, en sus mutuas relaciones, a partir de los argumentos de la primera teoría crítica, de Hannah Arendt, Giorgio Agamben y de la llamada filosofía de la diferencia, optando por pensar el problema de la experiencia en el sentido de poner en evidencia el hiato entre su expresividad y los lenguajes constitutivos de los saberes y las prácticas escolares ${ }^{5}$.

\footnotetext{
${ }^{2}$ Desse ponto de vista, por um lado, estudiosos como Jim Garrison (1999) e David Hansen (2004; 2008), ao reinterpretarem o pragmatismo de Dewey para elaborarem uma visão holística que compreende os saberes e as práticas escolares sob seus aspectos morais, intelectuais e artísticos, conferindo unidade à práxis educativa a partir das qualidades estéticas da experiência e de um pensamento reflexivo sustentado no instrumentalismo filosófico. Jover (2010); Jover, RuizValdepeñas y Thoilliez (2010); Bruno-Jofre y Jover (2011).

${ }^{3}$ Por outro, estudiosos como Trevisan (2000), Hermann (2005) e Dalbosco (2007), ao recuperarem a hermenêutica heideggeriana e a teoria crítica habermasiana, postulam que a experiência estética se constituiria em uma parte da razão comunicativa na qual deveria se sustentar a práxis educativa que, ao ser conceptualizada e integrada à pragmática da linguagem, poderia promover, em suas relações com a ética, a formação do homem.

${ }^{4}$ Embora esas perspectivas teóricas divirjam parcialmente em relação à articulação da experiência estética com as atitudes éticas e políticas e ao seu sentido na atualidade, com certa freqüência dialogam entre si em torno de pontos e proposições comuns, dentre eles sobressaindo o tema da experiência estética. Afinal, tais interpretações postulam apreender a experiência pelo conceito $e$ traduzir a sua dimensão estética em um gênero da linguagem que, em sua pragmática e na comunicação compreendida na práxis educativa, fosse capaz de compartilhá-la intersubjetivamente, por meio da conversação e do diálogo entre os participantes da comunidade. Assim, esses estudos postulam superar o hiato concernente a relação entre experiência e linguagem, concebendo que aquela pode ser traduzida em um dos gêneros desta e comunicada entre os participantes da comunidade.

${ }^{5}$ Estudiosos como Larrosa (2001; 2004), Bárcena (2004; 2006), Kohan (2003; 2008), Pagni (2010b), López (2009) e Gelamo (2009) vêm interpretando a inefabilidade da experiência, diante dos limites de sua apreensão pela linguagem, como um acontecimento que dá o que pensar e que 
Al abordar estas dos perspectivas teóricas, el presente proyecto intenta no sólo problematizar las respuestas epistemológicas relativas a la experiencia educativa, sino también, y principalmente, emitir una respuesta ética y política al problema de su empobrecimiento, con el subsiguiente declive de la transmisión y del pensar reflexivo en educación.

Una "respuesta ética", en la medida en que el problema exige, por parte de los sujetos de la acción pedagógica, una disposición de apertura y una actitud para enfrentar lo que irrumpe en la experiencia educativa como extraño y paradójico, como imprevisto y sorprendente (Audi, 2007); y una "respuesta política", en la medida en que supone una relación del sujeto de la educación consigo mismo, con un lenguaje y con una pragmática que organiza su acción en el mundo y que produce formas de subjetivación concordantes o en oposición a los modos actuales de gobierno y control educativo. Ambas respuestas implican un compromiso del sujeto de la educación con un pensar y un hacer vinculados a las continuidades $y$ rupturas necesarias entre la experiencia y sus lenguajes, la vida y la escuela, lo inefable y su comunicación, inaugurando una mirada que confiere cierta dignidad al oficio de enseñar y que, antes de limitarse a ser mera actividad técnicoprofesional, asume un modo de ser y de expresarse - desde una perspectiva estética, ética y existencial- en el tiempo presente.

Es justamente en relación con esta reflexión sobre estas dimensiones que los resultados de esta investigación pretender realizar una contribución frente a la impronta biopolítica del discurso pedagógico actualmente adquirida. En el fondo, el problema que nos planteamos y queremos explorar es tan antiguo como la pregunta que Sócrates ya formulara en el Protágoras. ¿es posible formar un buen ciudadano (arethé politiké), y sobre qué bases y condiciones? Sabemos que el diálogo platónico la pregunta queda sin respuesta; y tal vez deba permanecer así, incluso cuando la modernidad ha pretendido darle una respuesta positiva y afirmar, en consecuencia, que los Estados, por medio de las políticas educativas, tienen la obligación de educar a sus ciudadanos. Pero eso es algo que tanto las

remete os sujeitos da práxis educativa à infância do pensamento, entendida como a condição $e$ a possibilidade da sua emergência, como o silêncio que precede a irrupção da linguagem articulada. 
democracias como las dictaduras y los gobiernos totalitarios siempre pretendieron: las primeras, formando ciudadanos dotados con capacidad de juicio; las segundas, instruyendo súbditos obedientes y dóciles, y quizá, en ambos casos, mediante mecanismos equivalentes de adoctrinamiento político y procesos de conformismo social.

Esta pregunta nos parece fundamental, porque intuimos que, dentro del contexto biopolítico, existe lo que llamaremos un secuestro social de la experiencia de formación; o dicho en otros términos: asistimos a una reducción de la experiencia -estética, ética, política y existencial- de la educación a mera instrucción (y conformación social) de ciudadanos, un ciudadano normalizado y, en su vida cotidiana, también administrado. Bajo esas condiciones, le es retirado aquello que era tan estimado al hombre griego: su condición de bios polithikos. Así, lo que queremos saber es cómo en la contemporaneidad las (bio)políticas han limitado la propia condición humana del bios polítikos y condicionado su experiencia en el proceso de constitución de sí como sujeto de la educación.

\section{REFERENCIAS}

Adorno, Th. (1992) Minima Moralia: reflexões a partir da vida danificada, São Paulo: Editora Ática

Agamben, G. (1998) Homo Sacer, I. El poder soberano y la nuda vida, Valencia, Pre-Textos.

Agamben, G. (2001) Infancia e historia. Ensayo sobre la destrucción de la experiencia, Buenos Aires, Adriana Hidalgo Editora.

Agamben, G. (2008) Qu'est-ce que le contemporain?, Paris, Rivages.

Arendt, H. (1951) The Origins of Totalitarianism, Nueva York, Harcourt Brace Jovanovich.

Arendt, H. (1958) The Human Condition, Chicago, University of Chicago Press.

Arendt, H. (1961) Between Past and Future. Six Exercices in Political Thought, Nueva York, Viking Press.

Arendt, H. (1970) On Violence, Nueva York, Harcourt Brace Jovanovich.

Audi, P. (2007) Supériorité de l'éthique, Paris, Flammarion. 
Bárcena, F. (2004) El delirio de las palabras. Ensayo para una poética del comienzo. Barcelona, Herder

Bárcena, F. (2006a) La experiencia reflexiva en educación. Barcelona, Paidós.

Bárcena, F. (2006b) La re-politización de discurso cívico. Una mirada filosófica, en Revilla, F. (Coord.) Educación y ciudadanía. Valores para una sociedad democrática, Madrid: Biblioteca Nueva, pp. 76-96

Bárcena, F. (2006c) Hannah Arendt. Una filosofía de la natalidad, Barcelona, Herder.

Bárcena, F. (2006d) La sfinge muta. L'apprendimento del dolore dopo Auschwitz, Troino, Città Aperta.

Bárcena, F. (2008) Cuerpo, acontecimiento y educación, en G. Hoyos Vázquez,(coord.), Filosofía de la Educación, pp. 251-275. Madrid: Trotta

Bárcena, F. (2009a) Una pedagogía del mundo. Aproximación a la filosofía de la educación de Hannah Arendt, en Revista Anthropos, n 224, pp. 113-139.

Bárcena, F. y Mèlich, J-C. (2009b) L'educazione como evento ético. Natalità, narrazione, ospitalità, Roma, LAS Editrice.

Bárcena, F. (2011) The glow of fireflies. Philosophical essay for a recovery of educational experience, Revista de Innovación Educativa (México), 55, pp. 77-92.

Bárcena, F.; Asensio, J. M; Mèlich, J. C. (2001). Educación, vida humana y biopoder, en G. Vázquez, (ed.), Educación y calidad de vida, pp. 81-114. Madrid: Ed. Complutense

Benjamin, W. (2000a) Experiencia y pobreza, Obras Completas, Libro II, vol 1, Madrid, Abada editores.

Benjamin, W. (2000b) L'Oeuvre d'art a l'ère de sa reproductibilité technique, CEuvres, vol. III, París, Gallimard.

Benjamin, W. (1991) Paralipomènes et variantes des thèses sur le concept d'histoire, en Écrits Français, París, Gallimard.

Benjamin, W. (1982) Infancia en Berlín hacia 1900, Madrid, Alfaguara.

Bruno-Jofre, R. y Jover, G. (2011) The Readings of John Dewey's Work and the Intersection of Catholicism, en Bruno-Jofre, R. y Schriewer, J. (Eds.) The 
Global Reception of John Dewey's Thought. New York and London, Routledge, pp. 23-42.

Bustelo, E. (2007) El recreo de la infancia. Argumentos para otro comienzo, Buenos Aires, Siglo XXI Editores.

Braun, K. (2007), "Biopolitics and Temporality in Arendt and Foucault", Time Society 16,5 .

Brossat, A. (1996) L'épreuve du désastre. Le XX' siècle et les camps, Paris, Albin Michel.

Brossat, A. (2000) L'Animal démocratique. Notes sur la post-politique, Tours, Farrago.

Brossat, A. (2003) La démocratie inmunitaire, Paris, La Dispute.

Brossat, A. (2010) Droit à la vie? Paris, Éditions du Seuil.

Butler, J. (2006) Vida precaria (El poder del duelo y la violencia). Buenos Aires: Paidós.

Butler, J. (2010) Marcos de Guerra. Las vidas lloradas. Barcelona: Paidós.

Dalbosco, C. A. (2007) Pedagogia filosófica: cercanias de um diálogo, São Paulo: Paulinas.

Dean, M. (1999) Governability. Power and Rule in Modern Societies, London, Sage pub.

D’Hoest, F. y Bárcena, F. (2011) Las voces del acontecimiento. Un ensayo sobre el aprendizaje filosófico, Educaçao em Revista, 12/1, pp. 9-24

Didi-Huberman, G. (2009) Survivance des lucioles, Paris, Les Éditions de Minuit.

Esposito, R. (2003) Communitas. Origen y destino de la comunidad, Buenos Aires, Amorrortu editores

Esposito, R. (2005) Immunitas. Protección y negación de la vida, Buenos Aires, Amorrortu editores.

Esposito, R. (2006) Bios. Biopolitica y filosofia, Buenos Aires, Amorrortu editores.

Esposito, R. (2008) Communidad, inmunidad y biopolítica, Barcelona, Herder.

Esposito, R. (2009) Tercera persona. Política de la vida y filosofía de lo impersonal, Buenos Aires, Amorrortu editores.

Forti, S. (2008) El totalitarismo: trayectoria de una idea límite, Barcelona, Herder. 
Forti, S. (2006) "The Biopolitics of Souls. Racism, Nazism, and Plato". Political Theory 34: 9-32.

Foucault, M. (1976) Histoire de la sexualité: I. La volonté de savoir. Paris, Gallimard.

Foucault, M. (2006) Seguridad, territorio, población. Curso del Collège de France, 1977-1978, México, Fondo de Cultura Económica.

Foucault, M. (1997) Defender la Sociedad, Fondo de Cultura Económica, Buenos Aires, Argentina.

Foucault, M. (2002) Historia de la Sexualidad. 1- La Voluntad de Saber. Siglo XXI Editores, Buenos Aires, Argentina.

Foucault, M. (1997) Microfísica del poder, Ediciones de la Piqueta, Madrid, España.

Foucault, M. (2004) Naissance de la biopolitique. Cours au Collage de France (1978-1979). Gallimard-Seuil, Paris.

Foucault, M. (2010) El gobierno de sí y de los otros. Curso en el Collège de France, 1981-1982, México, 2010, F-C.E.

Gadamer, H-G, Verdad y Método, Salamanca, Sígueme.

Garrison, J. (1999) John Dewey's Theory of Practical Reasoning. Educational Philosophy and Theory.

Haraway, Donna, La biopolítica de los cuerpos posmodernos, en Ciencia, cyborgs y mujeres. La reinvención de la Naturaleza, Valencia, Cátedra, 1995.

Heller, A. y Feher, F, (1995) Biopolítica. La modernidad y la liberación del cuerpo, Barcelona, Península.

Hansen D. (2005) A poética no ensino. Educação em Revista: revista do Departamento de Administração e Supervisão Escolar da FFC/UNESP. Marília: UNESP Publicações, v. 1, n.6, p95-127.

Hansen, D. A (2008). Retratos de uma educação cosmopolita para a contemporaneidade. Educação \& Cultura Contemporânea: revista do mestrado em Educação. Rio de Janeiro: Universidade Estácio de Sá, Vol. 5, no. 9, [extra], p. 33-44. 
Hermann (2005) Ética e estética: a relação quase esquecida. Porto Alegre: EDIPUCRS, 2005 [Coleção Filosofia - 193].

Jay, M. (2005) Songs of Experience. Modern American and European Variations of a Universal Theme, Berkeley, 2005, University of California Press.

Jover, G. (2010) Readings of the pedagogy of John Dewey in Spain in the early twentieth century: reconciling pragmatism and transcendence, en BrunoJofre, R.; Johnston, J.S.; Jover, G. y Tröhler, D.: Democracy and the Intersection of Religion and Traditions. Montreal, McGill-Queen's University Press, pp. 79-130.

Jover, G.; Ruiz-Valdepeñas, M.A y Thoilliez, B. (2010) La obra de John Dewey en su tránsito por España durante el primer tercio del siglo XX. Dos ejemplos de recepción editorial. Edetania, 37, pp. 33-56.

Kohan, W. (2003) Infância. Entre Educação e Filosofia. Belo Horizonte: Editora Autêntica.

Kohan, W. (2008) Da filosofia da educação a uma educação da filosofia. Do estatuto à experiência: da quietude ao movimento. Educação \& Cultura Contemporânea: revista do mestrado em Educação. Rio de Janeiro: Universidade Estácio de Sá, Vol. 5, no. 9, [extra], p. 59-70.

Larrosa, J. Dar a palavra. Notas para uma dialógica da transmissão. In: Larrosa, J \& Skiliar, C. (orgs.). Habitantes de Babel: políticas e poéticas da diferença. Belo Horizonte: Editora Autêntica, 2001.

Larrosa, J. Algunas notas sobre la experiencia y sus lenguajes. In: Barbosa, R.L.L.(ORG.) Trajetórias e perspectivas da formação de educadores. São Paulo: Editora UNESP, 2004, p. 19-34.

Lazzarato, M. (2006) Los conceptos de vida y vivo en la sociedad de control, Buenos Aires, Tinta Limón.

Le Blanc, G. (2007) Les maladies de l'homme normal. Paris, Vrin.

Lewis, J (2006) The Schooll as an Exceptional Space: Rethinking Education from Perspective of the Biopedagogical, Educational Theory, 56:2, pp.159-176.

Lewis, J (2007) Biopolitical Utopianism in Educational Theory, Educational Philosophy and Theory, 39:7 
Lewis, J (2009) Education and the Immunization Paradigm, Studies in Philosophy of Educcation, 28, pp. 485-49.

Masschelein, J. (2001) The Discourse of Learning Society and the Loss of Childhood, Journal of Philosophy of Education, vol. 35, $\mathrm{n}^{\circ} 1$.

Mèlich, J-C. (2010) Ética de la compasión, Barcelona, Herder.

Mendiola Gonzalo, I. (2009) Rastros y rostros de la biopolítica, Barcelona, Anthropos.

Pagni, P. A. (2010ª) Infância, Arte de Governo Pedagógica e Cuidado de Si. Educação \& Realidade. Porto Alegre, v.35, n.3, p. 99-123.

Pagni, P. A. (2010b) Um lugar para a experiência e suas linguagens entre os saberes e práticas escolares: pensar a infância e o acontecimento na práxis educativa. In: pagni, P. A.; gelamo, R.P. Experiência, Educação $e$ Contemporaneidade. São Paulo/Marília: Cultura Acadêmica/Poïesis.

Pagni, P. y Gelamo, R. (2010) Experiência, Educação e Contemporaneidade. São Paulo/Marília: Cultura Acadêmica/Poiésis.

Pelloso Gelamo, R (2009)

Rancière, J. (1987) Ler maître ignorant, Paris, Payard.

Revault d'Allones, M. (1999) Le dépérissement de la politique. Généalogie d'un lieu commun. Paris, Aubier.

Stiegler, B. (2008) Prendre soin, de la jeunesse et des génèrations, Paris, Flammarion.

Stiegler, B. (2010) Ce qui fait que la vie vaut la peine d'être vécue, Paris, Flammarion.

Trevisan, A. L. (2000) Filosofia da Educação: mímesis e razão comunicativa, ljuí: Editora Unijuí.

Ugarte Pérez, J. (Comp.) (2005) La administración de la vida. Estudios biopolíticos. Barcelona, Anthropos.

Ugarte Pérez, J. (2006) Biopolítica. Un análisis de la cuestión, Claves de Razón Práctica, no 166 , pp. 76-82. 
Revista Sul-Americana de Filosofia e Educação - RESAFE

Vatter, M. (2007) "Arendt, Benjamin, and biopolitics," presentado en Hawaii University, Political Theory Workshop, abril 2007; a presentarse en Goethe Universität Frankfurt, Frankfurt, Alemania Political Theory Workshop, julio.

Vatter, M, (2006) "Natality and Biopolitics in Arendt", Revista de Ciencia Política, vol.26, n.2, pp. 137-159. 